\title{
Revascularization of a single-kidney occluded stent for renal salvage complicated by guide wire distal artery perforation and reperfusion injury
}

\author{
Angeliki Pastroma ${ }^{1 *}$ D, Stavros Spiliopoulos ${ }^{2}$, Konstantinos Palialexis ${ }^{2}$, Lazaros Reppas ${ }^{2}$ and Elias Brountzos ${ }^{2}$
}

\begin{abstract}
Background: The complexity of the case, including the rarefied simultaneous occurrence of complicationsiatrogenic, as well as reperfusion injury, invite reporting and publication.

Case presentation: A 39-year-old woman with a single-functioning left kidney, previous left renal artery stenting (RAS), and known hypercoagulopathy was hospitalized for flash pulmonary edema, elevated serum creatinine (9.7 $\mathrm{mg} / \mathrm{dl}$ ), and refractory hypertension. She was subsequently referred to our center [2] for endovascular treatment of acute renal ischemia (AKI) due to RAS occlusion.

Periprocedural complications of guide-wire arterial perforation and reperfusion injury resulted in life-threatening hemorrhage.

Conclusions: Following more than 48 h of hypoperfusion of the left kidney, revascularization of the thrombosed RAS was successfully attempted with selective, trans-catheter thrombolysis, and balloon angioplasty. Ultra-selective, nephron-sparing coil embolization was successfully performed. The patient's creatinine level decreased to $2.8 \mathrm{mg} / \mathrm{dl}$ at 12 days and to $1.5 \mathrm{mg} / \mathrm{dl}$ at 3 months. After 1 year of follow-up, the stent remains patent, and the patient is asymptomatic with stable renal function.
\end{abstract}

Keywords: Acute renal ischemia, Endovascular treatment, Balloon angioplasty, Trans-catheter arterial thrombolysis

\section{Background}

In the setting of renovascular hypertension (RVHT), anti-hypertensive drugs are indicated as initial treatment, while endovascular revascularization (RAS) is preserved for refractory cases, or as renal-salvage procedure in single-kidney cases with significant renal artery stenosis and deteriorating renal function [1]. As the American Heart Association/American College of Cardiology (AHA/ACC) guidelines do not match utterly those of the European Society of Cardiology (ESC) considering the indications for percutaneous revascularization, the

\footnotetext{
* Correspondence: angeliki.pastroma@gmail.com

'Department of Radiology, Metropolitan Hospital, Piraeus, Greece

Full list of author information is available at the end of the article
}

SCAI expert consensus concluded in 2014 that the candidates fit for renal artery stenting should present with cardiac disturbance syndromes, like flash pulmonary edema, as in our case, or acute coronary syndrome, hypertension resistant to medical treatment, not controlled by three or more medications at maximal doses, bilateral renal stenosis, or severe stenosis in a singlefunctioning kidney not controlled medically [2]. Positive outcomes following RAS have been reported in 96 to $100 \%$ of cases, with complications occurring in approximately $14 \%$, including renal artery rupture/dissection, distal embolization, contrast-induced nephropathy, renal bleeding, and stent thrombosis leading to acute kidney ischemia (AKI) [3-5]. Acute kidney ischemia is a medical emergency and should be treated immediately, as 
high rates of kidney loss have been reported in the literature after over $5 \mathrm{~h}$ of ischemia, despite revascularization [6].

\section{Case presentation}

A 39-year-old woman was emergently referred to our hospital with dyspnea, tachypnea, elevated serum creatinine and urea $(9.7 \mathrm{mg} / \mathrm{dl}$ and $173.5 \mathrm{mg} / \mathrm{dl}$ respectively), and refractory hypertension $(190 / 85 \mathrm{mmHg})$. The patient was initially admitted to another hospital for flash pulmonary edema and deterioration of her previously medically controlled hypertension The patient was known with hypercoagulopathy (positive for Factor V G1691A (FV-Leiden) mutation) and single-functioning left kidney with previously treated stenotic left renal artery by balloonexpandable stent placement (12 months ago). The right renal artery was occluded, according to digital subtraction angiography (DSA). She was regularly followed up by a nephrologist, with a relatively stable serum creatinine level at $1.3 \mathrm{mg} / \mathrm{dl}$, and was systematically prescribed clonidine, nifedipine, and acetylsalicylic acid (ASA).

Prior to her referral to our hospital's Interventional Radiology Unit, a duplex ultrasound (DUS) was performed, which demonstrated a markedly increased flow velocity within the proximal left renal artery, with a maximal velocity above $350 \mathrm{~cm} / \mathrm{s}$, along with aliasing artefacts within the stent, which appeared to be occluded. The intrarenal resistance index was also markedly reduced. The right kidney was morphologically diminished in size with concomitant loss of cortical thickness. The diagnosis of over $48 \mathrm{~h}$, acute renal ischemia (ARI) was made and taking into consideration the radiological findings, in addition to the patient's young age and gravely impaired renal function, a multidisciplinary team concluded that urgent endovascular treatment was appropriate. The patient was treated with intravenous heparin infusion during hospitalization, which was stopped $12 \mathrm{~h}$ prior endovascular treatment.

Following skin antisepsis at the right groin with povidone-iodine solution scrub, administration of prophylactic intravenous antibiotics (cefazolin) and local anesthesia, a right common femoral artery access was obtained using a $6 \mathrm{~F}$ sheath. "A bolus intraarterial dose of unfractionated heparin 5000 IU was administrated through the sheath. Standard heparinized saline was used during the procedure." Angiography with a pigtail catheter within the aorta at the level of the renal arteries depicted occlusion of the right renal artery and occlusion of the stent at the origin of the left renal artery (Fig. 1 a). However, the renal artery distal to the stent remained perfused by collaterals and appeared patent at delayed DSA images (Fig. 1 b). The occluded stent was successfully catheterized using a 5Fr USL 2 catheter (Cordis, USA) and in-stent thrombus was depicted (Fig. $1 \mathrm{c}$ ). A bolus dose of rtPA $5 \mathrm{mg}$ was administrated through the catheter within the thrombus. The lesion was then surpassed using a 0.018 in. guide wire (V18, Boston Scientific, USA), and angioplasty was performed with a $5.5 \times 40 \mathrm{~mm}$, low-profile, mono-rail, angioplasty balloon catheter (Submarine Rapido; Invatec S.p.A, Italy) (Fig. 2 a). During balloon angioplasty, the patient experience left flank pain, and the looped V18 guide wire was noted to be erroneously advanced within the distal renal branches (Fig. 2 b, c). During the final DSA, extravasation of contrast media was depicted at the site of guide wire manipulation, but also at multiple sites of the kidney irrelevant to

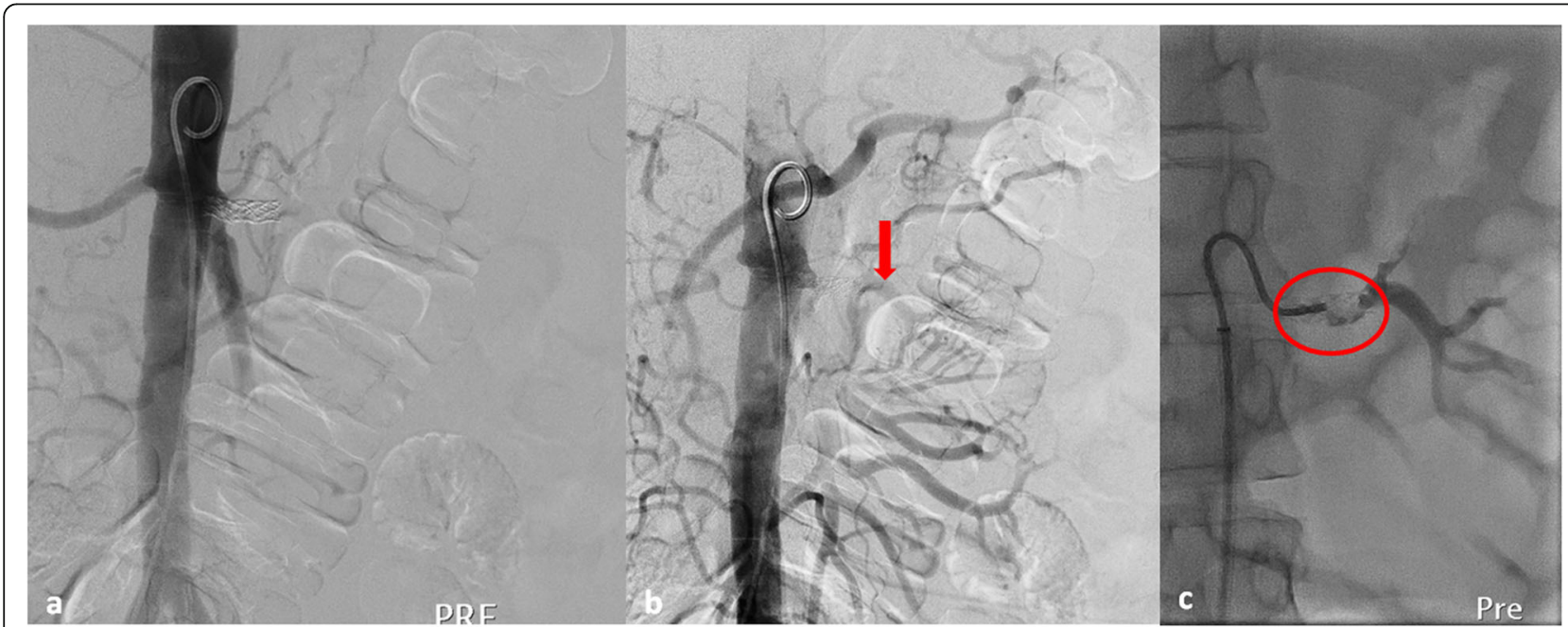

Fig. 1 a DSA at the level of the renal arteries demonstrating bilateral renal artery occlusion. b Delayed phase demonstrating perfusion of the distal renal artery (arrow) from collateral network. c Selective catheterization of the occluded stent and contrast infusion demonstrating in-stent thrombosis with characteristic contrast media feeling defects (circle) 


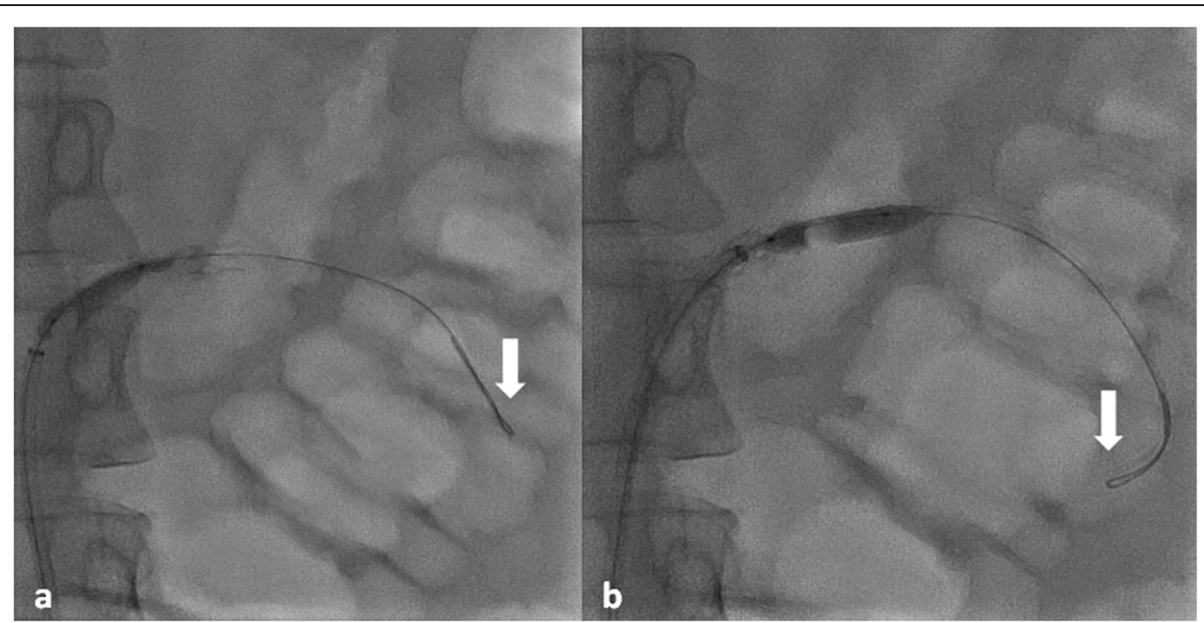

Fig. 2 a Balloon angioplasty of the origin of the renal artery and proximal stent segment, followed by 2nd balloon inflation more distally within the stent and the renal artery (b). Note the accidental distal migration of the guide wire (arrows)

guide-wire distal migration and therefore attributed to reperfusion injury (Fig. 3 a). The patient's flank pain increased remarkably, the systolic blood pressure dropped from $190 \mathrm{mmHg}$ to $120 \mathrm{mmHg}$, and a significant sub capsular hematoma was noted during parenchymal phase DSA (Fig. 3 a). Nephron-sparing, ultra-selective, coil embolization was performed (Fig. 3 b, c) using a microcatheter (Progreat, Terumo, Japan) and by deploying two pushable $3 \times 40 \mathrm{~mm}$ and $3 \times 70 \mathrm{~mm}$ micro-coils (Nester, Cook, USA). However, following embolization extravasation was still noted, at the site of guide wire trauma while all the other sites of extravasation were not depicted (Fig. 3 c). The feeding branch was catheterized with the microcatheter and another pushable $3 \times 140 \mathrm{~mm}$ micro-coil was deployed (Fig. 3 d). The vessel appeared occluded at fluoroscopy with minimal contrast infusion, and final DSA was not performed as the operator was convinced that the bleeding was stopped and further contrast media administration was deemed unnecessary and harmful to the already compromised renal function. Supportive conservative treatment was initialized, and the patient was transfused with three units of blood and remained hemodynamically stable.

After 12 days, the patient was discharged with normal blood pressure, serum creatinine level of $2.8 \mathrm{mg} / \mathrm{dl}$, urea $115.2 \mathrm{mg} / \mathrm{dl}$, and hemoglobin $10.2 \mathrm{mg} / \mathrm{dl}$ along with electrolytes within normal range. After 1 month, the creatinine level was $2.1 \mathrm{mg} / \mathrm{dl}$, which after 3 months returned to nearly baseline value of $1.5 \mathrm{mg} / \mathrm{dl}$. The patient was prescribed dual antiplatelet therapy (clopidogrel $75 \mathrm{mg}$ and aspirin $100 \mathrm{mg}$ once daily) for 1 year and was scheduled for a strict clinical and DUS follow-up at 1, 3, 6, and 12 months. After 1 year of follow-up, the stent remains patent and the patient is asymptomatic with stable renal function.

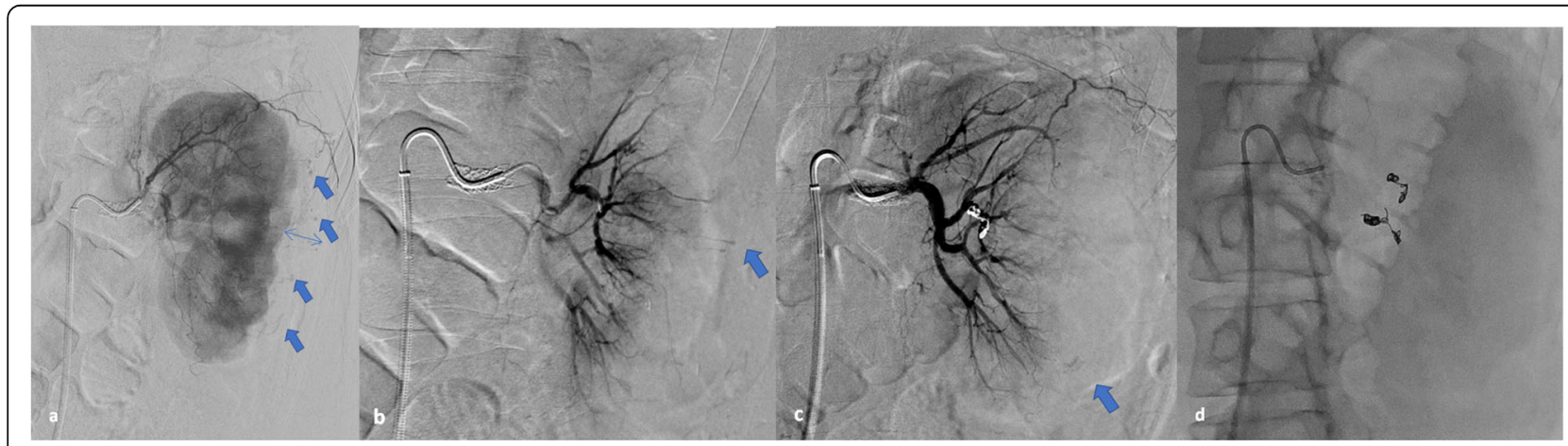

Fig. 3 a Final DSA demonstrating multiple foci of active extravasation at the upper, middle, and lower pole of the kidney (arrowhead) with corresponding large subcapsular hematoma (double arrow). b Selective DSA following the first coil embolization of one renal artery branch depicted a remaining site of active bleeding at the point of guide-wire distal migration (arrow). c Note that all other foci of extravasation are not depicted anymore. $\mathbf{d}$ Fluoroscopic image following second coil embolization of the more caudal renal artery branch 


\section{Discussion}

We describe the case of a young adult with thrombophilia, suffering from right functional nephrectomy and significant left renal artery stenosis, who underwent initial RAS due to both uncontrollable hypertension and deteriorating renal function. The procedure was clinically successful, as creatinine levels nearly normalized and hypertension was controlled with two hypertensive drugs. However, in-stent thrombosis occurred 12 months after stent placement, despite ongoing single antiplatelet therapy, and was correlated to the patient's hypercoagulable state. Perhaps single antiplatelet therapy was not adequate in this case, considering the underlying hypercoagulopathy. For that reason, following revascularization, the patient was prescribed with dual antiplatelet therapy for at least 1 year, despite the fact that high level of evidence and guidelines concerning the type and duration of antithrombotic therapy in such cases do not exist. The patient suffered from symptomatic AKI causing a hypertensive crisis and flash pulmonary edema, due to long lasting in-stent thrombosis. According to the literature, acute renal ischemia lasting for over 5 hours has been correlated with kidney loss despite revascularization [6]. Nevertheless, successful delayed endovascular revascularization of ARI even $72 \mathrm{~h}$ after the occlusive event has been sporadically reported in the literature and therefore, revascularization could be attempted in selected cases [7]. The patient's young age, excellent performance status, and the presence of minor perfusion within the renal parenchyma at DUS set the indication for the attempt of endovascular renal-salvage revascularization, despite the long-standing ischemia.

Bilateral renal artery occlusion was confirmed by initial DSA, while following selective catheterization of the left renal artery, the presence of in-stent thrombus was indubitably demonstrated by the characteristic pattern of contrast filling defect. Nevertheless, acute thrombosis in the ambit of gradually progressive renal artery stent restenosis could not be ruled out. The fact that collateral filling of the renal artery, distal to the occluded stent, was noted at delayed phase DSA and was probably the main factor contributing to the preservation of parenchymal perfusion after $48 \mathrm{~h}$ of ALI and ultimately renal salvage. Direct stenting was not attempted as it was thought that the stent would not surpass the occlusion without balloon pre-dilation, especially in the presence of significant restenosis. Low-profile balloon angioplasty was successfully performed, following trans-catheter thrombolysis. However, during the last balloon advancement within the stent, the patient experienced sudden left flank pain and the V18 guide wire was noted to be more distally advanced than previously causing vessel perforation and severe hemorrhage. It is known that the specific guide-wire, if not correctly contained, can perforate small caliber vessels. However, the V18 was thought to provide the adequate support and lesion "cross-ability" required in this case. Moreover, following renal artery reperfusion, multiple bleeding sites were noted at the upper, mid, and lower pole of the distal renal parenchyma. As the guide-wire never approached the specific areas, bleeding was attributed to reperfusion injury. Sudden restoration of blood flow in previously stenotic or occluded renal arteries may trigger kidney injury [1]. Hyperperfusion of ischemic tissue with oxygenated blood leads to immense oxidative stress, activating an inflammatory cascade [5]. Analogous to the cerebral hyperperfusion syndrome following revascularization of the internal carotid artery, the dominant hypothesis considering the mechanism of injury is loss of autoregulation $[8,9]$. While the pathophysiology remains unclear, it is suggested that post-procedural hyperperfusion results in augmented pressure in the distal renal bed, rendering it susceptible to hemorrhage [9].

\section{Conclusion}

To our knowledge, this is the first case report of iatrogenic severe bleeding due to both accidental guide-wire perforation and reperfusion injury attributed to the long-standing acute renal ischemia. Despite the fact that the exact time of in-stent thrombosis could not be determined (as in the vast majority of ARI cases reported in the literature), this case demonstrates the value of revascularization attempt in selected cases, especially when there is strong suspicion that renal perfusion is marginally preserved by collateral flow.

\section{Abbreviations \\ RAS: Renal artery stenting; AKI: Acute renal ischemia; RVHT: Renovascular hypertension; AHA/ACC: American Heart Association/American College of Cardiology; ESC: European Society of Cardiology; DSA: Digital subtraction angiography; ASA: Acetylsalicylic acid; DUS: Duplex ultrasound; ARI: Acute renal ischemia}

\section{Acknowledgements}

Not applicable

\section{Authors' contributions}

AP: data curation, writing original draft-editing. SS: conceptualization, writing review-editing, procedure performance. KP: image curation, procedure performance. LR: procedure performance, patient follow-up. EB: supervision, validation, procedure performance. Written consent was obtained from the participant prior to publication.

All authors have read and approved the manuscript before submission.

\section{Funding}

None

Availability of data and materials

Data and material used during the current study are available from the corresponding author upon reasonable request.

Ethics approval and consent to participate

The patient included in this study/case report gave written informed consent to undergo the medical procedure, as well as to participate in this research. 


\section{Consent for publication}

The patient included in this study/case report gave written informed consent to publish their data contained within this study.

\section{Competing interests}

None

\section{Author details}

'Department of Radiology, Metropolitan Hospital, Piraeus, Greece. ${ }^{2}$ 2nd Department of Radiology, Interventional Radiology Unit, University of Athens, University General Hospital "Attikon", Athens, Greece.

Received: 18 June 2020 Accepted: 9 August 2020

Published online: 07 September 2020

\section{References}

1. Textor SC (2013) Renovascular hypertension: is there still a role for stent revascularization? Current Opinion in Nephrology and Hypertension. Curr Opin Nephrol Hypertens 22(5):525

2. Parikh SA et al (2014) SCAl expert consensus statement for renal artery stenting appropriate use. Catheter Cardiovasc Interv 84(7):1163-1171 Axelrod, David J., et al. "Guide wire perforation leading to fatal perirenal hemorrhage from transcortical collaterals after renal artery stent placement." Journal of vascular and interventional radiology 15.9 (2004): 985-987

3. Axelrod DJ et al (2004) Guide wire perforation leading to fatal perirenal hemorrhage from transcortical collaterals after renal artery stent placement. J Vasc Interv Radiol 15(9):985-987

4. Ivanovic $V$ et al (2003) Renal artery stent placement: complications at a single tertiary care center. J Vasc Interv Radiol 14(2):217-225

5. Malek M, Nematbakhsh M (2015) Renal ischemia/reperfusion injury; from pathophysiology to treatment. J Renal Inj Prev 4(2):20

6. Jawas A, Abu-Zidan FM (2008) Management algorithm for complete blunt renal artery occlusion in multiple trauma patients: case series. Int J Surg 6(4): 317-322

7. Arabi M, Vellody R, Cho K (2011) Acute renal artery occlusion with prolonged renal ischemia: a case of successful treatment with stent placement and catheter-directed thrombolysis. J Clin Imaging Sci 1:11

8. Kang KP et al Renal subcapsular hematoma: a consequence of reperfusion injury of long standing renal artery stenosis. Electrolyte Blood Press 5(2): 2007, 136-2139

9. Allison RP, Belli AM, Chun JY et al (2014) Renal arterial hemorrhage following renal artery stenting. Managing Common Interventional Radiology Complications. Springer, London, pp 183-189

\section{Publisher's Note}

Springer Nature remains neutral with regard to jurisdictional claims in published maps and institutional affiliations.

\section{Submit your manuscript to a SpringerOpen ${ }^{\circ}$ journal and benefit from:}

- Convenient online submission

- Rigorous peer review

- Open access: articles freely available online

High visibility within the field

- Retaining the copyright to your article 